\title{
SEGURIDAD ALIMENTARIA EN MÉXICO: REFORMA DEL ESTADO Y MODERNIZACIÓN ADMINISTRATIVA
}

\section{FOOD SECURITY IN MEXICO: STATE REFORM AND ADMINISTRATIVE MODERNIZATION}

\author{
Yessika Mabel Cepeda Arellano ${ }^{1}$ \\ Martha Elena Báez Martínez ${ }^{2}$
}

Recibido: 2021-09-18 / Revisado: 2021-10-20 / Aceptado: 2021-11-25 / Publicado: 2022-01-01

Forma sugerida de citar: Cepeda-Arellano, Y. M. y Báez-Martínez, M. E. (2022). Seguridad alimentaria en México: Reforma del Estado y modernización administrativa. Retos de la Ciencia. 6(12). 62-73. https://doi.org/10.53877/rc.6.12.20220101.06

\section{RESUMEN}

El presente trabajo tiene por objetivo el dar respuesta a la pregunta: ¿De qué manera la estructura y el funcionamiento del Estado y la Administración Pública se relacionan con la Seguridad Alimentaria en México? Ello se logrará a través de un enfoque de investigación mixto/holístico que permitirá llevar a cabo el análisis de las Reformas del Estado Mexicano y de los procesos más relevantes implementados para la Modernización Administrativa en materia de Seguridad Alimentaria. Así como también, se podrá observar que las acciones emprendidas han generado que la producción interna de alimentos se incremente y que la balanza agroalimentaria se encuentre en una etapa superavitaria, lo cual da como resultado que exista el acceso físico de alimentos al interior del país, tal y como lo plantean los preceptos de la Seguridad Alimentaria.

Palabras clave: seguridad alimentaria, reforma del Estado, modernización administrativa.

\section{ABSTRACT}

The objective of this work is to answer the question: In what way are the structure and operation of the State and Public Administration related to Food Security in Mexico?

\footnotetext{
${ }^{1}$ Doctora en Administración Pública. Catedrática en la Facultad de Derecho y Ciencias Sociales de la Benemérita Universidad Autónoma de Puebla, México. E-mail: yessika.cepeda@correo.buap.mx / ORCID https://orcid.org/0000-0003-0253-2783

2 Doctora en Administración Pública. Catedrática en la Facultad de Contaduría Pública de la Benemérita Universidad Autónoma de Puebla. México. E-mail: martha.baez@correo.buap.mx / ORCID https://orcid.org/00000003-2394-7750
} 
This will be achieved through the analysis of the Mexican State Reforms and the most relevant processes implemented for Administrative Modernization in the area of Food Safety. As well, it will be possible to observe the articulation of the mechanisms to guarantee Food Security that has tended to supply domestic demand through the importation of food products from international markets, mainly from the United States, with the Free Trade Agreement of North America, today a Treaty between Mexico, the United States and Canada, leaving aside the enormous production capacity that the field has in Mexico.

Keywords: food security, state reform, administrative modernization

\section{INTRODUCCIÓN}

Ante un mundo dinámico, donde el peso que jugaban las naciones hegemónicas de Estados Unidos de América y la Unión de Repúblicas Socialistas Soviéticas (URSS), los grandes triunfadores después de la Segunda Guerra Mundial, generaban tensiones entre los países del globo durante el periodo de la llamada Guerra Fría, en la cual se buscaba la supremacía de un único país poderoso en todas las áreas, tanto político, económico, militar y sobre todo ideológicamente hablando, debido a que el capitalismo y el socialismo buscaban consolidarse en el mayor número de naciones para fomentar sus preceptos.

Los postulados del Estado de Bienestar a nivel internacional comienzan a ver su desencanto a partir de los años 70, donde la lógica de la intervención del Estado ve su agotamiento a través de los déficits presupuestarios que las naciones tenían, principalmente latinoamericanas. Ello, derivado de las enormes cantidades de dinero que debían de destinar al cumplimiento de los servicios y demandas sociales, al endeudamiento al que debían recurrir los países, y al enorme tamaño de la maquinaria burocrática que atendía a la razón de la creación de empleos.

Este panorama, aunado a la cercanía de México con los Estados Unidos, con la siempre estrecha relación histórica y los intereses latentes hacia el territorio latinoamericano, llevó a los Estados Unidos y su modelo neoliberal a establecerse en México, después de haberlo instaurado ya en el país de Chile, por citar un ejemplo. La intención principal era alejar las funciones del Estado en el funcionamiento económico, a través de la reorientación de las políticas económicas y el redimensionar el aparato gubernamental, lo que cambiaría la relación entre el actuar del Estado y la sociedad.

Esto generó el inicio de una serie de reformas en el país para lograrlo y sobre todo adecuarlo al contexto que se vivía, encaminado ahora a la desarticulación de un modelo de bienestar hacia un modelo neoliberal. Para Francisco Valdés Ugalde (Valdés, 1993), “...la reforma de la intervención estatal en la economía se basa en la convicción de que el crecimiento económico depende de la capacidad competitiva de cada economía nacional para situarse en el intercambio mundial sin recurrir a medidas proteccionistas internas o externas". Mediante la reforma de la intervención del Estado se fue perdiendo poco a poco las medidas proteccionistas dejando a ciertas áreas desprotegidas, donde el Estado tenía una fuerte injerencia para su funcionamiento, afectando a las naciones.

No únicamente las condiciones internacionales fomentaron que México adoptara un modelo neoliberal como lo dictaban los Estados Unidos, a través de sus organismos financieros internacionales. Ello, debido a que al interior la mala administración pública y la toma de decisión de un grupúsculo ocasionó al país caer en una de las peores crisis que había atravesado en la historia, lo cual desembocaría 
en que se adquiriera una deuda casi imposible de pagar, pero necesaria para reactivar las aristas que el Estado debía cubrir y dirigir. Dicha deuda se adquirió al momento de firmar un acuerdo de facilidad ampliada con una entidad internacional como lo es el Fondo Monetario Internacional, pues había un déficit presupuestario que hacía incapaz al Estado de operar a favor de los ciudadanos, entre otros temas.

Entre las causas principales que ocasionaron la crisis de 1982 fue el modelo de la economía que implementó México basado en la petrolización que dirigió a la política económica de 1976 a 1982, donde se daba prioridad al sector petrolero motivando a una cada día mayor dependencia de la exportación del petróleo al extranjero y de los precios del petróleo a nivel internacional.

De acuerdo con Paulina Chávez Ramírez (Chávez, 1996) “...la crisis que vivió México en 1982 marcó un punto de inflexión en la economía mexicana por dos razones: porque se interrumpieron cuatro décadas de crecimiento y la inflación alcanzó niveles que no se habían registrado antes, y porque se dio un giro drástico en el enfoque del desarrollo económico por parte del gobierno".

La crisis económica que vivió México ocasionó que en noviembre de 1982 se firmara la tan conocida Carta de Intención con el Fondo Monetario Internacional y al tomar posesión del cargo el presidente Miguel de la Madrid Hurtado, el 1 de diciembre de 1982, se pone en ejecución el Programa Inmediato de Recuperación Económica (PIRE), donde se instrumentaban las medidas establecidas y necesarias en dicha Carta para su gradual implementación.

No obstante, las Reformas y la Modernización Administrativa que el Estado Mexicano ha llevado a cabo no han generado cambios significativos para garantizar la Seguridad Alimentaria de los ciudadanos, hecho que ha fomentado el abandono del campo y una seria interdependencia en cuanto a los compromisos internacionales que se han firmado con naciones extranjeras.

\section{METODOLOGÍA}

En el presente ensayo se llevó a cabo un trabajo de gabinete, basado en una metodología cualitativa, donde se revisaron las acciones prioritarias implementadas en México para garantizar la Seguridad Alimentaria, así como también se tomó en cuenta una metodología cuantitativa, debido a que se buscaron datos que establecieran la situación de la Seguridad Alimentaria en México, así como la situación de la producción de alimentos que se refleja a través de la balanza comercial agroalimentaria. Por ende, este trabajo refleja una metodología mixta de investigación.

\section{RESULTADOS/DISCUSIÓN}

A nivel internacional, el tema de la Soberanía y Seguridad Alimentaria ha logrado posicionarse como un eje trascendental para el desarrollo y bienestar humano. Entre los antecedentes más relevantes, se encuentra la Organización de las Naciones Unidas para la Alimentación y la Agricultura (FAO, por sus siglas en inglés), como organismo especializado de las Naciones Unidas, fundada en 1945, en el cual quedaba claro el objetivo general de lograr un mundo donde imperara la seguridad alimentaria elevando los niveles de nutrición, mejorando la productividad agrícola, las condiciones de la población rural, y contribuyendo a la expansión de la economía mundial. 
A partir de 1948, en la Declaración Universal de los Derechos Humanos, en donde el concepto de seguridad alimentaria aparece en el escenario internacional como un derecho fundamental plasmado en el artículo 25, donde se menciona que toda persona tiene derecho a un nivel de vida adecuado que le asegure, así como a su familia, la salud y el bienestar, y en especial la alimentación, el vestido, la vivienda, la asistencia médica y los servicios sociales necesario. Sin embargo, este concepto tomó fuerza en los años 70 del siglo XX, cuando los precios internacionales de los alimentos básicos se fueron al alza y provocaron que países como África, Asia y América Latina enfrentaran la escasez de alimentos y hambrunas. Ello aunado al incremento de los precios del petróleo y el de los fertilizantes.

A principios de los años setenta se hizo evidente la existencia de una severa crisis en la producción agrícola. El abastecimiento suficiente de alimentos básicos, en especial el de granos, fue el problema central y la expresión más aguda de la crisis. Hasta 1970, y desde mediados de los cincuenta, el país había sido básicamente autosuficiente, sin embargo, en los setenta, el abasto interno empezó a depender en forma creciente de las importaciones que llegaron a representar entre una cuarta y una tercera parte del consumo nacional (Warman, 2002).

Cabe mencionar, que la capacidad de autosuficiencia alimentaria se dio básicamente por una serie de instrumentos de política agrícola que le daban certeza. Entre dichos instrumentos se pueden citar los precios de garantía, los cuales surgen en 1953 con el objetivo de incrementar la producción agrícola para con ello poder garantizar la producción de alimentos y materias primas a través de subsidios directos a la producción, comercialización y transformación, mediante precios subvencionados. El primer cultivo que se estableció su precio de garantía fue el fríjol, posteriormente, el maíz y el trigo. En 1970, ya había 12 productos que estaban sujetos a la dinámica de los precios de garantía.

La instancia encargada de elaborar los análisis técnicos para establecer los precios de garantía en el país fue la Comisión Coordinadora del Sector Agropecuario, la cual se integraba por la Secretaría de Agricultura y Ganadería, de la Presidencia, y de los Recursos Hidráulicos, de los bancos Agrícola, Ejidal y Agropecuario, la Aseguradora Agrícola, la Productora Nacional de Semillas, así como también de la Compañía Nacional de Subsistencias Populares (CONASUPO).

Los precios de garantía permitían que los campesinos garantizaran la compra de su producción con precios favorables, lo cual permitía un nivel de vida digno, y una autosuficiencia en la producción de alimentos sin la necesidad de importarlos del exterior.

Sin embargo, entre 1965 y 1972, se empieza a relegar al sector agrícola a causa de la política económica del gobierno federal que lo dejaba en desventaja frente al sector industrial. Con esto se generó un déficit en la producción de alimentos en relación con la población, promoviendo la necesidad de optar por la compra de importaciones para cubrir las necesidades.

Para corregir las tendencias negativas provocadas por la escasa participación de los productos mexicanos ante sus competidores extranjeros, se implementaron el Sistema Alimentario Mexicano (SAM) y la Ley de Fomento Agropecuario, "conjunto de disposiciones estatales orientados a promover el crecimiento de la producción agropecuaria y mejorar el ingresos de los productores" (Olmedo, 1991) con las cuales el sector agropecuario y rural recibió una importante transferencia de recursos a principios de la década de los ochenta, la cual no se repitió hasta la puesta en marcha del Programa de Apoyos Directos al Campo (PROCAMPO). 
EI SAM fue creado por la oficina de asesores presidenciales que dedicó dos años al estudio sistemático del campo, el Sistema Alimentario Mexicano fue anunciado por José López Portillo el 18 de marzo de 1980, dentro del marco de las celebraciones de la expropiación petrolera y junto con la decisión de que México no ingresaría en el GATT.

En el contexto estatal tiene singular importancia la implementación del Sistema Alimentario Mexicano en 1979, encargado de impulsar una política de apoyo a la producción cerealera, basada en una concepción distinta a la que había imperado tradicionalmente. EI SAM tenía como propósito elevar los rendimientos agrícolas a través de la distribución selectiva de los recursos, orientados hacia campesinos ubicados en tierras de buen temporal mayores de 5 hectáreas. EI SAM fue visto como el programa más ambicioso para respaldar el viejo compromiso del Estado con la autosuficiencia alimentaria nacional, así como también fue visto como el último mecanismo de intervención fuerte del Estado en el sector agrícola.

El reto, para los encargados de un programa tan grande e innovador como lo fue el SAM, surge al intentar asegurar que los recursos de que dispone el gobierno subsidien en la mayor medida posible a los segmentos de población que más los necesiten, y en la menor medida posible a los grupos que más tienen, es decir, para reducir el desperdicio de recursos hay que basarse en criterios claros de progresividad. Y esto, en el caso de México de principios de la década de 1980, tenía implicaciones políticas inaceptables (Hewitt de Alcántara, 2007).

EI SAM dejó, mediante, su experiencia dos elecciones generales: 1) demostró la capacidad de respuesta del sector agrícola mexicano para producir suficientes granos básicos para alimentar al país; y 2) demostró que los recursos agrícolas asignados difícilmente llegarían a los campesinos en ausencia de canales institucionales democráticos que obligaran a rendir cuentas a los aparatos del Estado dirigidos a atender la producción agropecuaria (Fox, 1990).

La política económica instrumentada en el sexenio de Miguel de la Madrid se apega a los lineamientos del ajuste estructural: desregulación de la inversión extranjera y bolsa de valores, privatización gradual y por etapas de sectores estratégicos, ajuste fiscal, pago puntual del servicio de la deuda externa, entre otros. Pero uno de los temas que aparece de manera recurrente en las cartas de intención que periódicamente firma el gobierno federal con el FMI, sobre todo aparecen con nitidez de 1983 en adelante, es la desregulación de la agricultura, privatización gradual de las empresas públicas del sector agropecuario, eliminación de subsidios agrícolas, apertura comercial, eliminación gradual de las cuotas de importación y baja y posterior eliminación de aranceles.

Con la entrada de México al Acuerdo General de Aranceles y Comercio (GATT) en 1986, se estableció el compromiso de liberalización gradual de la agricultura mexicana, pero gracias a las reservas del protocolo de adhesión introducidas por el equipo negociador se introdujeron ciertos candados que preservaron facultades regulatorias, mecanismos de protección agrícola y el derecho a aplicar subsidios.

Sin embargo, a partir de 1989, la presión librecambista de los EUA se incrementa y el gobierno de Carlos Salinas tiene una debilidad institucional producto de su falta de legitimidad. Esto, unido a la necesidad de contar con flujos de capital especulativo como medio para una precaria estabilización de la economía, origina que México inicie negociaciones para el Tratado de Libre Comercio de América del Norte (TLCAN) en condiciones de debilidad. Por el contrario, la agenda estadounidense estaba perfectamente clara. De hecho, era una versión actualizada de la posición que durante años mantuvieron, misma que estaba presente en los acuerdos con el FMI y 
en las propuestas de la delegación estadounidense a la Ronda Uruguay del GATT, iniciada en 1986 y culminada ocho años después en Marruecos.

En 1992, se lleva a cabo en México la Reforma Agraria que reformó el Artículo 27 Constitucional, el cual se publicó el 6 de enero en el Diario Oficial de la Federación, así como también en el mismo año, pero el 26 de febrero se publica la Ley Agraria que es la encargada de regular las modificaciones del artículo en cuestión.

Tabla 1:

Balanza Comercial Agropecuaria y Agroalimentaria (Millones de dólares)

\begin{tabular}{|c|l|l|l|l|l|l|l|}
\hline \multirow{2}{*}{ Año } & Saldo & \multicolumn{4}{|c|}{ Exportaciones } & \multicolumn{3}{c|}{ Importaciones } \\
\cline { 2 - 8 } & Total & Total & Agropecuario & Agroalimentario & Total & Agropecuario & Agroalimentario \\
\hline 1980 & -895 & 2,289 & 1,517 & 772 & 3,184 & 2,014 & 1,170 \\
\hline 1985 & 41 & 2,152 & 1,401 & 751 & 2,111 & 1,603 & 508 \\
\hline 1990 & $-1,535$ & 3,206 & 2,111 & 1,095 & 4,741 & 2,062 & 2,679 \\
\hline 1991 & $-1,044$ & 3,712 & 2,291 & 1,421 & 4,756 & 2,121 & 2,635 \\
\hline 1992 & $-2,764$ & 3,417 & 2,052 & 1,365 & 6,181 & 2,845 & 3,336 \\
\hline 1993 & $-1,934$ & 4,039 & 2,449 & 1,590 & 5,973 & 2,617 & 3,356 \\
\hline 1994 & $-2,822$ & 4,512 & 2,616 & 1,896 & 7,334 & 3,345 & 3,989 \\
\hline 1995 & 1,189 & 6,432 & 3,903 & 2,529 & 5,243 & 2,627 & 2,616 \\
\hline 1996 & $-1,455$ & 6,315 & 3,385 & 2,930 & 7,770 & 4,654 & 3,116 \\
\hline
\end{tabular}

Fuente: Elaboración propia con datos obtenidos del Banco de México. (Banco de México, 2021).

La apertura de la economía nacional por la que México atravesaba en los años 90, la cual marcó cambios importantes en la forma de intervenir por parte del gobierno, obligó a que el grupo en el poder implementara las medidas necesarias para que el sector agropecuario entrara en la era de la modernización, evitando su rezago y hasta su gradual desaparición por falta de apoyos económicos y estratégicos, modificando el esquema tradicional de apoyos para el campo.

En el cuadro 1, se puede observar, que México tenía un déficit en su balanza comercial, debido a que importaba más de lo que exportaba. Sólo en el año 1985 y 1995 registró un saldo favorable de 41 y 1,189 millones de dólares respectivamente. En 1992 y 1994, se presenta los déficits más altos de 2,764 y 2,822. En general, la tabla confirma que se importa más en el sector agrícola a causa de incapacidad de producción en el país.

El deterioro en los precios desalentó el incremento de la producción comercial y provocó que las unidades de producción empresariales abandonaran estos cultivos ya que era imposible obtener una ganancia o ésta era demasiado baja en comparación con otras actividades, así como también, era casi imposible competir con los bajos precios de sus competidores en el extranjero.

En el marco del Programa Nacional de Modernización del Campo (1990-1994) y a la incorporación de México a organismos económicos internacionales con el Acuerdo General sobre Aranceles Aduaneros y Comercio (GATT) hoy Organización Mundial del Comercio (OMC) y el Tratado de Libre Comercio de América del Norte (TLCAN), el PROCAMPO fue instrumentado a finales del ciclo agrícola de 1994.

El propósito primordial del PROCAMPO era mejorar el nivel de ingreso de las familias que conforman las áreas rurales, sobre todo a los productores que destinan su producción al autoconsumo y no a la comercialización de esta. EI PROCAMPO fue 
creado bajo la idea de colaborar con otros objetivos básicos a lo largo de su implementación, los cuales son:

- Coadyuvar al incremento de la capacidad de capitalización de las unidades de producción.

- Promover la capitalización del sector productivo rural,

- Frenar la degradación del medio ambiente, contribuyendo a la recuperación y conservación de bosques y selvas.

- Convertir superficies productivas en actividades de rentabilidad mayor,

- Regularizar la tenencia de la tierra en cumplimiento de lo dispuesto por el Artículo 27 constitucional.

El Programa de Apoyos Directos al Campo (PROCAMPO) es un programa creado en 1993 por el Gobierno Federal para beneficiar al sector agrícola, el cual consiste en transferir recursos fiscales en apoyo al ingreso de los productores rurales. El PROCAMPO, a diferencia de otros programas de apoyo al campo, consistía en un apoyo anual continuado y de largo plazo, debido a que tendrá una duración de quince años, contados a partir de 1994.

Básicamente, el PROCAMPO respondía a la necesidad de la economía nacional de dar pie a la apertura ante los mercados globales. Con ello, se dio paso a la modernización del campo mexicano, razón por la cuál a finales de 1993, el PROCAMPO emprendió su operación, periodo que marca el inicio de labores del año agrícola Otoño-Invierno 1993/1994.

El Banco Mundial, a través del Informe sobre pobreza y hambre, incorpora dos indicadores para el análisis de la seguridad alimentaria que son: la inseguridad alimentaria crónica y la inseguridad alimentaria transitoria. Es para 1996, que se llevó a cabo la Cumbre Mundial de Alimentación, donde se estableció una noción integral del concepto de seguridad alimentaria, la cual es la siguiente: "Existe seguridad alimentaria cuando todas las personas tienen en todo momento acceso físico y económico a suficientes alimentos inocuos y nutritivos para satisfacer sus necesidades alimenticias y sus preferencias en cuanto a los alimentos a fin de llevar una vida activa y sana." (FAO, 2014).

Asimismo, en la Cumbre Mundial de Alimentación, se aprobó la Declaración de Roma y el Plan de Acción de la Cumbre, en las cuales se plasman los objetivos para garantizar la seguridad alimentaria.

En el caso de México, la Soberanía y Seguridad Alimentaria se colocó como temática prioritaria en la política nacional, a raíz de la crisis de los precios de los alimentos, y la incapacidad del mercado de satisfacer la producción interna de alimentos, lo que orilló al Estado a comprar productos estratégicos del exterior. Esto factores provocaron una disminución de los ingresos de la población y un considerable aumento en los niveles de inseguridad alimentaria.

Para Eduardo García Maynez (Garcia, 2009) el derecho Alimentario es el “... conjunto de normas, principios usos y costumbres que tienen por objeto regular la protección de los recursos agropecuarios, así como su transformación, comercialización y consumo, con la finalidad de preservar la salud nutricional." El Estado no sólo debe encargarse de proveer el abasto de alimentos a su población, sino que también, dichos alimentos deben de ser de calidad, para con ello, promover una alimentación nutricional acorde con los requerimientos calóricos y fomentar la salud a nivel nacional. 
De acuerdo con Edmundo Hernández-Vela Salgado (Hernandez - Vela, 2002, p. 1086) la Seguridad Alimentaria se define como el:

[...] conjunto de estrategias, políticas, normas medidas y acciones tendientes a garantizar el abasto y el acceso permanentes, incluso en previsión de contingencias ambientales o de cualquier otra índole, en cantidad, variedad, composición, combinación, calidad y precios suficientes, adecuado y satisfactorios, de los alimentos y complementos requeridos para su cabal salud y bienestar y su pleno desarrollo y evolución, tanto individual como social.

Para algunos autores, los conceptos de Soberanía Alimentaria y Seguridad Alimentaria tienden a utilizarse deliberadamente, llegando hasta confundir los objetivos de cada una de ellas. Es debido a esto, que, para la presente investigación, se deben tener claro que son nociones que trabajan en conjunto, y que se dan fuerza entre sí.

Según el Panorama de la Seguridad Alimentaria y Nutricional de la FAO, "La erradicación de la inseguridad alimentaria requiere un rediseño de la política y estrategias orientadas a tal fin, en un proceso participativo que considere la multidimensionalidad de la seguridad alimentaria, la institucionalidad que asegure la coordinación y la coherencia de las políticas sectoriales y la propuesta de los recursos indispensables" (FAO, 2014).

Es debido a lo anterior, que el gobierno de México implementó como fase primordial para garantizar la Soberanía y la Seguridad Alimentaria en el 2011, el establecer constitucionalmente el derecho al Acceso a la Alimentación. En el Artículo $4^{\circ}$ Constitucional se llevaron a cabo 3 reformas, en las cuales el 13 de octubre de 2011, quedó asentado que "toda persona tiene derecho a la alimentación nutritiva, suficiente y de calidad. El Estado lo garantizará".

En el año 2013, el gobierno mexicano implementó la Cruzada Nacional Contra el Hambre, con el objetivo de dar impulso, a nivel nacional, al eje temático contemplado dentro del Plan Nacional de Desarrollo, para que 7.4 millones de personas superen su condición de pobreza extrema y carencia alimentaria. Esta iniciativa está integrada por 70 programas federales, y dio inicio con su primera etapa dentro de 400 municipios a nivel nacional. Además, buscó cumplir con las entidades especializadas a nivel internacional, como, por ejemplo, los Objetivos del Desarrollo del Milenio, hoy Objetivos de Desarrollo Sostenible.

A pesar de este recorrido histórico, donde se describen las reformas del Estado y la implementación de mecanismos más relevantes que promovían la modernización de la administración pública en la noción de la Seguridad Alimentaria, en 2018 el Consejo Nacional de Evaluación de la Política de Desarrollo Social (CONEVAL) reportó que existen 52.4 millones de personas en situación de pobreza, lo que equivale al $41.9 \%$ de la población que registran un promedio de 2.2 carencias sociales. En cuanto a la carencia alimentaria en el país se registran 25.5 millones de mexicanos, es decir el $20.4 \%$ de la población total.

En la Tabla 2, se observa que la inseguridad alimentaria en el país aún afecta a un porcentaje importante de la población que ha variado a lo largo del tiempo y que más ha incidido en la población con inseguridad alimentaria severa. 
Tabla 2:

Porcentaje de personas en los componentes de carencia por acceso a la alimentación nutritiva y de calidad 2008-2020.

\begin{tabular}{|c|c|c|c|c|c|c|c|}
\hline \multirow{2}{*}{ Indicadores } & \multicolumn{7}{|c|}{ Porcentaje } \\
\hline & 2008 & 2010 & 2012 & 2014 & 2016 & 2018 & 2020 \\
\hline \multicolumn{8}{|l|}{$\begin{array}{l}\text { Carencia por acceso a la } \\
\text { alimentación }^{3}\end{array}$} \\
\hline \multirow{4}{*}{\begin{tabular}{ll}
\multicolumn{2}{l}{ Seguridad alimentaria } \\
Inseguridad & alimentaria \\
leve & \\
Inseguridad & alimentaria \\
moderada & \\
Inseguridad & alimentaria \\
severa & \\
\end{tabular}} & 53.9 & 55.7 & 56.1 & 57.5 & 59.3 & 60.3 & 57.8 \\
\hline & 24.4 & 19.5 & 20.6 & 19.1 & 20.6 & 19.3 & 21.5 \\
\hline & 12.8 & 14.0 & 13.7 & 13.4 & 11.9 & 12.1 & 12.7 \\
\hline & 8.9 & 10.8 & 9.7 & 9.9 & 8.1 & 8.3 & 8.1 \\
\hline
\end{tabular}

En la gestión gubernamental del actual presidente de México, Andrés Manuel López Obrador, se tiene contemplado de forma más amplia la temática de la Seguridad Alimentaria, iniciando con el Decreto Presidencial con fecha de 18 de enero de 2019 que da origen a un organismo descentralizado de la Secretaría de Agricultura y Desarrollo Rural con personalidad y recursos propios denominado SEGALMEX.

Con estos programas se pretende rescatar al sector del campo, así como también para fomentar la autosuficiencia alimentaria y dejar de depender de los productos de origen extranjero.

\section{Figura 1:}

SEGALMEX como eje de Política Pública Alimentaria en México

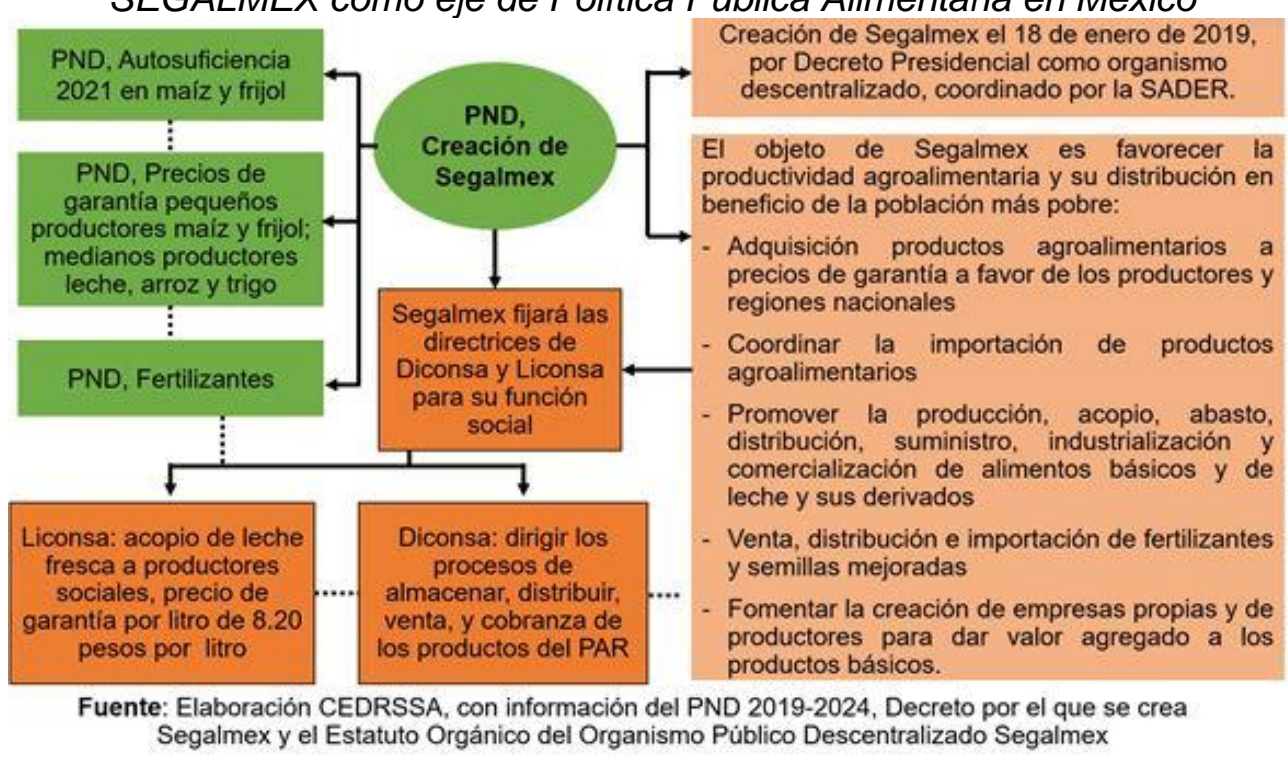

Es innegable que la producción agroalimentaria y agroindustrial en México, a partir del año 2015, se encuentra en una etapa superavitaria, como se aprecia en la gráfica 1 , debido a que se exportan un mayor número de productos de los que se están importando del mercado internacional. Esto permite entender que la producción 
nacional de alimentos está cumpliendo con los preceptos de la Seguridad Alimentaria en cuanto al abasto de la demanda interna.

En el periodo de 1993 a 2014, las condiciones en México eran diferentes, debido a que se encontraba en un déficit de alimentos, es decir se importaba más de lo que se exportaba, de acuerdo con la balanza agroalimentaria y agroindustrial.

No obstante, México en la actualidad se coloca a nivel internacional como el primer y décimo importador de maíz y trigo en grano respectivamente. Por ello, el gobierno del presidente Andrés Manuel López Obrador plantea la necesidad de orientar al país hacia una Soberanía Alimentaria más allá de contar con una Seguridad Alimentaria al interior del territorio sin tener que comprar productos de los mercados internacionales de alimentos a través del fortalecimiento del campo.

\section{Gráfica 1:}

México: Balanza agropecuaria y agroindustrial 1993-2021 (junio)

Miles de dólares

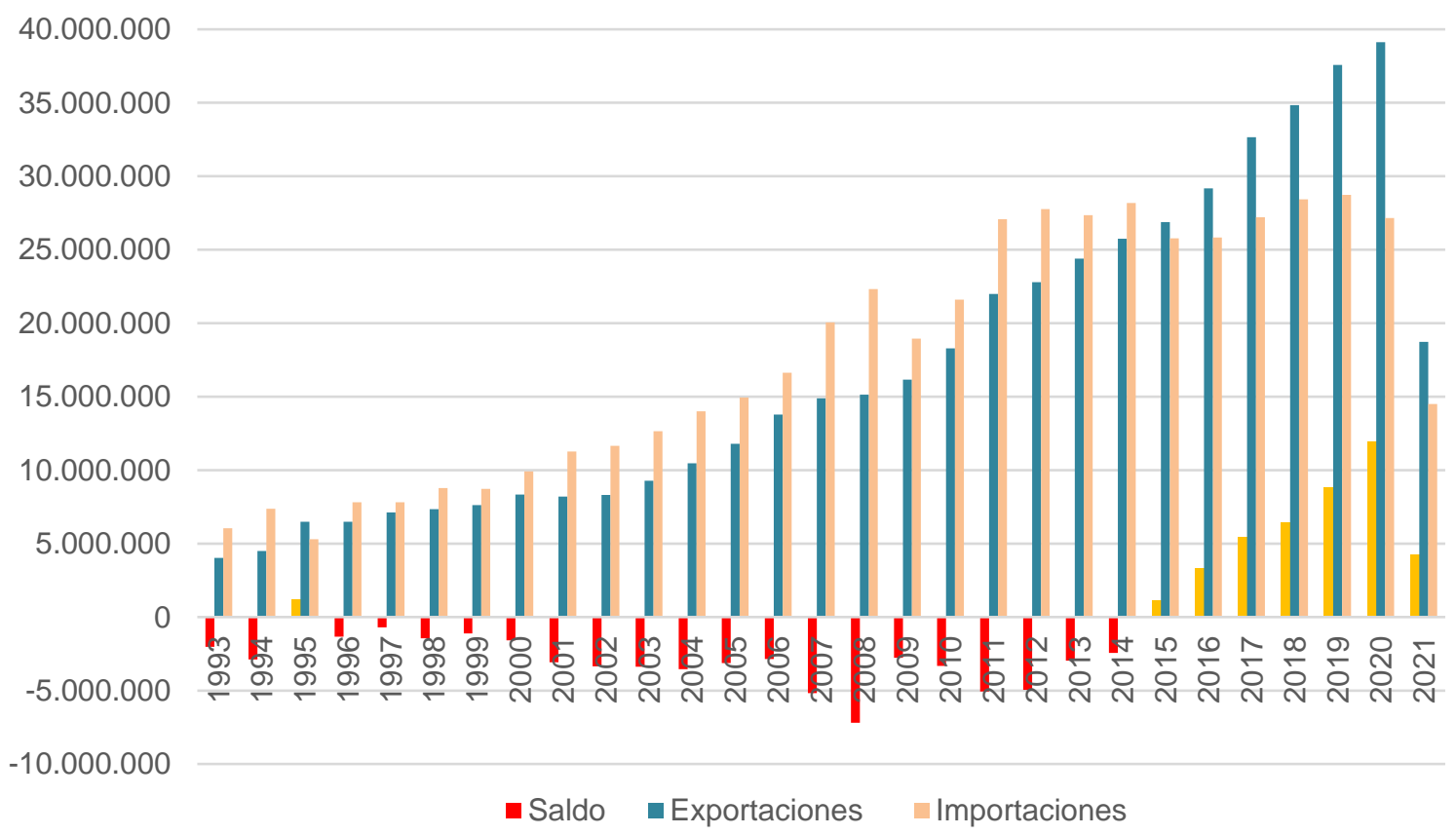

Fuente: Banxico, 2021.

\section{CONCLUSIONES}

A lo largo de esta investigación se revisaron las grandes transformaciones a las que se sometió a la administración pública y a los ciudadanos por el hecho de haber dejado de lado al Estado Benefactor por dar paso al modelo neoliberal.

Se observó que se han creado una serie de mecanismos gubernamentales bajo la lógica de garantizar el derecho a la alimentación mediante la Seguridad Alimentaria. Sin embargo, se han establecido programas alineados con el contexto internacional como lo son el Programa Nacional de Modernización del Campo y el PROCAMPO, que buscaba resarcir el abandono de los subsidios al campo que dictaban los organismos financieros internacionales en la dinámica neoliberal. 
Asimismo, se implementaron políticas sociales que buscaban resarcir la problemática del hambre y la pobreza como lo fue PRONASOL, CONASUPO, la Cruzada Nacional Contra el Hambre, principalmente.

Actualmente, en el gobierno del presidente Andrés Manuel López Obrador se implementó como programa prioritario la búsqueda de la Soberanía Alimentaria y la atención al campo. No obstante, será indispensable esperar los resultados del programa, así como los efectos que dejará la pandemia primordialmente a los sistemas alimentarios.

No hay que dejar de lado que, a nivel global existen factores que ocasionan la inseguridad alimentaria, tal como es el cambio climático, la contaminación y erosión de los suelos, los precios internacionales de los productos alimenticios, la calidad de los alimentos, los precios del petróleo, la insistencia por apoyar cultivos establecidos no suficientes ni variados, el ingreso y las oportunidades laborales. Razón por la cual, se tiende a buscar mecanismos para garantizar la Seguridad y Soberanía Alimentaria a través de estrategias que incentiven la participación de las unidades familiares.

\section{REFERENCIAS BIBLIOGRÁFICAS}

Banco de México. (17 de octubre de 2021). www.banxico.org.mx. Obtenido de https://www.banxico.org.mx/Sielnternet/consultarDirectoriolnternetAction.do?s ector $=1$ \&accion $=$ consultarCuadroAnalitico\&idCuadro=CA187\&locale $=e s$

Chávez, P. (1996). Las cartas de intención y las políticas de estabilización y ajuste estructural de México: 1982 - 1994. En Premio Anual de Investigación Económica Maestro Jesús Silva Herzog. (pág. 172). México: Universidad Nacional autónima de México, Instituto de Investigaciones Económicas.

Constitución Política de los Estados Unidos Mexicanos. Recuperado de http://www.diputados.gob.mx/LeyesBiblio/pdf/1_280521.pdf 16 de octubre de 20201.

FAO. (2014). Panorama de la Seguridad Alimentaria y Nutricional en América Latina $y$ el Caribe. (2013). Panorama de la Seguridad Alimentaria y Nutricional en México 2013, FAO-ONU-SAGARPA-SEDESOL-Instituto Nacional de Salud Pública.

Fox, J. (1990). La dinámica del cambio en el sistema alimentario mexicano. 1980 1982. En Historia de la cuestión agraria mexicana. Los tiempos de la crisis. Primera Parte (págs. 170-1982). México, D.F.: Siglo XXI - CEHAM.

Garcia, E. (2009). En C. Valero, El derecho a la alimentación y la soberanía alimentaria (El caso mexicano). (pág. 19). México, D.F.: Comité del Centro de Estudios de Derecho e investigaciones Parlamentarias.

Hernandez - Vela, E. (2002). Diccionario de Política Internacional. México, D.F.: Porrúa.

Hewitt de Alcántara, C. (. (2007). Ensayo sobre los obstáculos al desarrollo rural en México. Retrospectiva y prospectiva. Revista desacatos, núm. 25., 79-100.

Olmedo, R. (1991). El Sistema Alimentario Mexicano y la Ley de Fomento Agropecuario. Nueva Antropología. revista de Ciencias Sociales, Núm. 17. Instituto de Investigacuones Jurídicas de la UNAM. México, D.F.

ORTIZ Wadgywar, A. (1988). El fracaso neoliberal en México, 6 años de Fondo Monetarismo (1982-1988), Editorial Nuestro Tiempo, México, pp. 135.

Valdés, F. (1993). Concepto y estrategia de la Reforma del Estado. revista Mexicana de Sociología, 315-338. 
Warman, A. (2002). La reforma agraria mexicana: una visión de largo plazo. Obtenido de http://www.fao.org/3/j0415t/j0415t09.htm\#TopOfPage 